\title{
Spermogramme de béliers Peuls bicolores et Touaregs : influence du type génétique et de la saison
}

\author{
M. Issa ${ }^{1}$ A. Yenikoye ${ }^{1}$ H. Marichatou ${ }^{1}$ M. Banoin ${ }^{1}$
}

\section{Mots-clés}

Ovin - Mouton Peul - Mouton Touareg - Sperme - Fertilité - Niger.

\section{INTRODUCTION}

L'élevage des ovins constitue une importante source de revenu pour une grande partie de la population nigérienne. Les moutons fournissent une part non négligeable des protéines animales nécessaires aux populations rurales et citadines et jouent un rôle socioculturel important lors des cérémonies de mariage, baptême, décès, etc. (9).

1. Université Abdou Moumouni, faculté d'Agronomie, BP 10960, Niamey, Niger Tél : 733238 ; fax : 733238 ; e-mail : cresa@intnet.ne ; issamoumouni@yahoo.fr
Pour répondre à la demande sans cesse croissante en produits animaux du fait de la démographie galopante, il est nécessaire d'intensifier la production. Ceci impose une meilleure connaissance des performances zootechniques des animaux et des facteurs liés au milieu qui les affectent. La mise au point de méthodes et techniques pour tirer le meilleur profit des capacités de reproduction des animaux implique une connaissance aussi rigoureuse que possible de la fonction de reproduction.

C'est ainsi que des études antérieures ont permis de constater un faible taux de reproduction annuel chez la brebis Peule bicolore en milieu réel, pouvant être imputable aux brebis ou à une variation saisonnière de la fertilité du bélier (24). Malheureusement, on dispose de peu d'informations sur les capacités reproductrices du 
mâle $(14,15)$. Ces études ont précisé les caractéristiques morphologiques du sperme de béliers de race Peule et Touareg, mais les effets des différents facteurs de l'environnement sur ces paramètres en relation avec la saison ne sont pas connus.

L'objectif de ce travail a été de déterminer la production spermatique ainsi que les caractéristiques de fertilité du sperme et leur variation saisonnière chez deux races de béliers provenant de deux systèmes écologiques différents.

\section{MATERIEL ET METHODES}

L'expérience a été réalisée de janvier 1996 à décembre 1998, au parc de la faculté d'Agronomie de Niamey (18 $30^{\circ}$ de lat. N, $2^{\circ} 08^{\prime}$ de long. E, $216 \mathrm{~m}$ d'altitude).

\section{Données climatiques}

Les paramètres climatiques ci-après ont été enregistrés au cours de la période expérimentale (figure 1) :

- la température moyenne a été minimale en décembre et en janvier $\left(25,7^{\circ} \mathrm{C}\right)$, avec deux pics, l'un en mai $\left(34,4{ }^{\circ} \mathrm{C}\right)$ et l'autre en octobre $\left(32^{\circ} \mathrm{C}\right)$;

- l'amplitude thermique a été la plus faible en juin $\left(8,4{ }^{\circ} \mathrm{C}\right)$ et maximale en décembre $\left(17,7^{\circ} \mathrm{C}\right)$;

- le taux d'humidité relative a été minimal en mars $(15,1$ p. 100) et maximal en octobre $(44,7$ p. 100$)$;

- la pluviométrie moyenne à Niamey durant les années 1996, 1997, 1998 a été respectivement de 548,3 mm, 411,6 mm et 816,6 mm avec une saison sèche longue de huit mois (octobre à mai) et une saison humide courte de quatre mois (juin à septembre) ;

- la photopériode a été minimale en décembre (11 h 03) et maximale en mai (12 h 08) (22).

\section{Animaux}

Deux races de béliers provenant de deux systèmes écologiques et d'élevage différents ont été utilisées : six béliers de race Peule bicolore provenant des marchés situés dans le sud-ouest du pays en zone soudano-sahélienne et cinq béliers de race Touareg provenant du nord du pays correspondant à la zone sahélo-saharienne. Ces

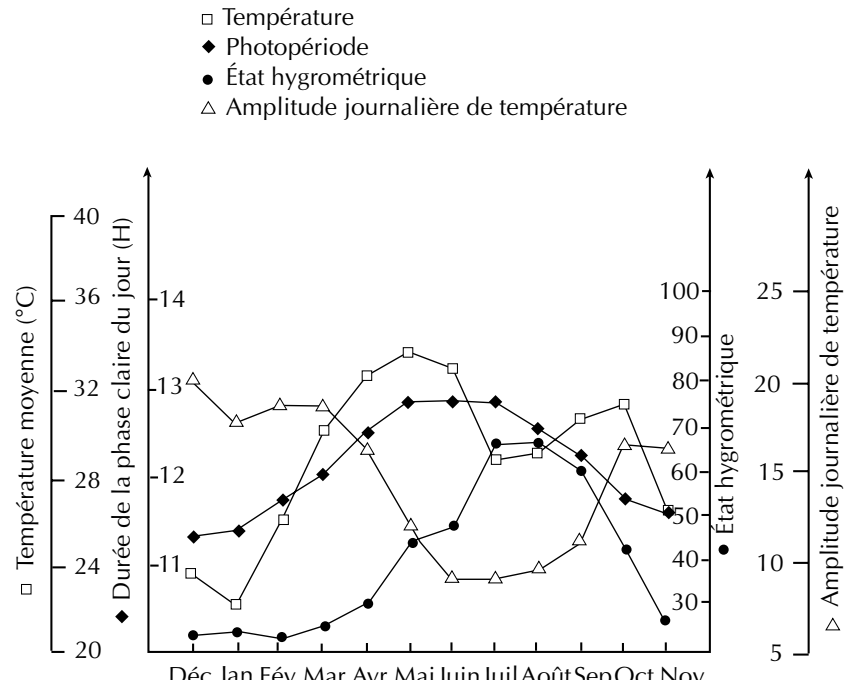

Figure 1 : variations mensuelles des facteurs climatiques à Niamey (Yenikoye A., 1986, thèse Doct., université de Tours, France). animaux ont été régulièrement déparasités et vaccinés contre la pasteurellose, les charbons et la peste des petits ruminants.

Au début de l'expérience, les béliers étaient âgés de 1 à 2 ans et pesaient de 27 à $46 \mathrm{~kg}$. Leur régime alimentaire était composé de paille de riz, de son de riz, de graines de coton, d'oligo-éléments sous forme de pierre à lécher et d'eau ad libitum. La croissance testiculaire étant fonction du poids corporel, les animaux ont été pesés chaque mois.

\section{Collecte du sperme}

Le sperme a été collecté au vagin artificiel à l'aide d'une brebis en chaleur immobilisée. Le sperme de chaque bélier a été prélevé une fois par mois à raison de deux éjaculations par séance de collecte. Les analyses du sperme ont été effectuées sur le volume final de sperme des deux éjaculats.

\section{Dilution du sperme}

Le sperme collecté a été dilué au 1/401 dans du sérum physiologique formolé $(\mathrm{NaCl} 9 \%$ + formol 1 p. 100). Cette préparation a servi, d'une part, au comptage des spermatozoïdes sur la cellule de Mallasez, d'autre part, à la détermination de la densité optique $(\lambda=520 \eta \mathrm{m})$. Ces opérations visent à déterminer la concentration du sperme en spermatozoïdes.

Le sperme a aussi été dilué au 1/5 dans du tampon phosphate. Cette préparation a permis de réaliser un frottis coloré à l'éosine/nigrosine, en vue de déterminer les taux de spermatozoïdes morts et des différentes anomalies.

\section{Analyses du sperme}

\section{Examen immédiat}

Après la deuxième éjaculation, le volume final a été noté à travers le tube collecteur gradué. Une goutte du sperme collecté a été ensuite observée au microscope photonique au faible grossissement afin d'apprécier la motilité massale notée de 0 à 5 (0= im-mobilité totale, $5=$ mobilité rapide avec tourbillons).

\section{Détermination de la concentration en spermatozoüdes du sperme}

A partir de 36 échantillons de sperme de chacune des deux races, une droite de régression, traduisant l'évolution de la concentration en spermatozoïdes (déterminée par comptage sur cellule de Mallasez) en fonction de la densité optique (lue au spectrocolorimètre à 520 ๆm) du sperme dilué, a été établie. Ceci a permis par la suite de déterminer la concentration en spermatozoïdes de chaque semence après lecture de la densité optique du sperme dilué et à partir de l'équation de la droite de régression.

\section{Détermination du taux de spermatozoüdes morts et anormaux}

Les frottis préparés ont été observés au microscope photonique (x 400) pour déterminer sur 150 spermatozoïdes le nombre de morts et le nombre présentant l'une des anomalies suivantes :

- anomalie de la tête (AT) ; tête piriforme, microcéphalie, acrosome endommagé ;

- décapité ou tête sans flagelle (SD) ;

- présence de gouttelette cytoplasmique en position proximale (GP) ;

- présence de gouttelette en position distale (GD) ;

- anomalie du flagelle (AF) ; flagelle replié, ou pièce intermédiaire coudée.

Lorsqu'un spermatozoïde a présenté deux anomalies, seule l'anomalie la plus grave a été recensée selon l'ordre décroissant suivant : AT, SD, GP, GD, AF (4). 


\section{Analyses statistiques}

L'analyse de variance à trois critères de classification a été utilisée pour étudier les effets race, saison, année sur les paramètres mesurés. Une transformation angulaire des pourcentages (angle $=$ arcsinus $\sqrt{ }$ pourcentage) de spermatozoïdes morts et anormaux a été effectuée avant l'analyse de variance, afin de normaliser les données (19). Le test de la plus petite différence significative au niveau $\alpha=0,05$ a été utilisé pour comparer deux à deux les différentes moyennes d'une même variable. Les analyses ont été effectuées à l'aide du logiciel Sas (Statistical Analysis System).

\section{RESULTATS}

Les moyennes des différents paramètres étudiés durant les trois années chez les deux races de béliers sont indiquées dans le tableau I.

\section{Variations saisonnières du poids corporel}

Au cours de l'année, le poids moyen des béliers a varié de manière significative $(p<0,05)$ chez les deux races. La saison hivernale (juillet-août) n'a pas été favorable à une prise de poids des animaux, alors que la saison sèche fraîche (novembre-décembre) a été favorable (figure 2).

\section{Variations avec la saison et l'âge des paramètres spermatiques}

\section{Volume de sperme}

La différence de volume de sperme éjaculé entre les deux races n'a pas été significative $(\mathrm{p}>0,05)$. Toutefois, en juillet et août les béliers Touaregs ont eu un volume moyen de sperme significativement plus élevé que celui des béliers Peuls : respectivement 1,74 $\pm 0,32 \mathrm{ml}$ et $2,07 \pm 0,39 \mathrm{ml}$ contre $1,5 \pm 0,31 \mathrm{ml}$ et $1,57 \pm 0,32 \mathrm{ml}$.

La saison a affecté de manière significative $(\mathrm{p}<0,05)$ le volume moyen de sperme seulement chez les béliers Peuls bicolores. Durant l'hivernage (juillet-août) les béliers Peuls ont produit un volume moyen de sperme significativement plus faible $(\mathrm{p}<0,05)$ qu'en avril, septembre, octobre et novembre (figure 3).

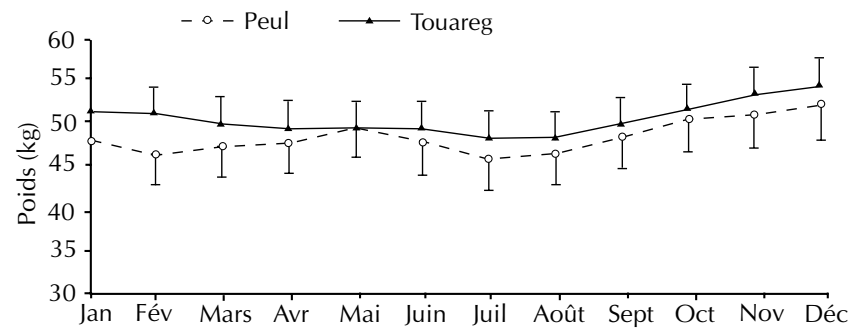

Figure 2 : variations mensuelles du poids corporel moyen chez les deux races de béliers (moyenne \pm écart-type).

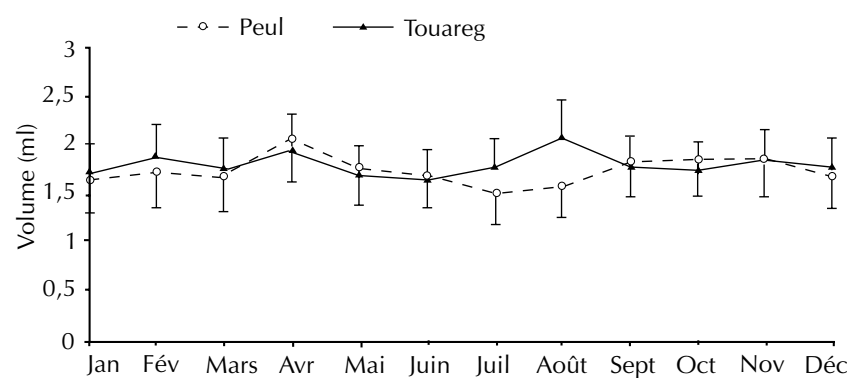

Figure 3 : variations mensuelles du volume moyen de sperme chez les deux races de béliers (moyenne \pm écarttype).

Chez les deux races de moutons, le volume moyen de sperme éjaculé en 1997 a été significativement plus élevé $(\mathrm{p}<0,05)$ que ceux de 1996 et 1998. Les résultats obtenus ont été chez les béliers Peuls de 1,93 ml en 1997 contre $1,58 \mathrm{ml}$ en 1996 et $1,66 \mathrm{ml}$ en 1998, et chez les béliers Touaregs de $2 \mathrm{ml}$ en 1997 contre $1,56 \mathrm{ml}$ en 1996 et $1,75 \mathrm{ml}$ en 1998 .

\section{Motilité massale}

Chez les deux races, la motilité massale moyenne s'est affaiblie de façon significative pendant la saison sèche fraîche (novembre à février) par rapport aux autres mois. Chez les béliers Peuls,

\section{Tableau I}

Effectif, poids et spermogramme des béliers Peuls bicolores et Touaregs étudiés pendant trois ans (deux éjaculats consécutifs une fois par mois)

\begin{tabular}{|c|c|c|c|}
\hline & Peuls bicolores & Touaregs & Différence significative \\
\hline Effectif & 6 & 5 & \\
\hline Poids vif moyen (kg) & $47,75 \pm 3,75$ & $49,98 \pm 2,99$ & * \\
\hline Volume (ml) & $1,73 \pm 0,36$ & $1,78 \pm 0,33$ & ns \\
\hline Motilité massale & $4 \pm 0,61$ & $3 \pm 0,49$ & $*$ \\
\hline Concentration (x $\left.10^{6} \mathrm{spz} / \mathrm{ml}\right)$ & $4265 \pm 762$ & $4953 \pm 583$ & $* *$ \\
\hline Nb. moyen de spz totaux $\left(x 10^{6}\right)$ & $7469 \pm 2382$ & $8877 \pm 2323$ & * \\
\hline Spz morts (\%) & $12 \pm 10$ & $19 \pm 19$ & * \\
\hline Anomalies totales (\%) & $13 \pm 12$ & $17 \pm 11$ & $* *$ \\
\hline $\begin{array}{l}* \text { Significatif, } \mathrm{p}<0,05 \\
* * \text { Significatif, } \mathrm{p}<0,01 \\
\mathrm{~s}=\text { non significatif } \\
\mathrm{pz}=\text { spermatozoïdes }\end{array}$ & & & \\
\hline
\end{tabular}


la motilité a été plus élevée de mai à octobre. Chez les béliers Touaregs, la motilité a été la plus élevée en juin-juillet et en septembre-octobre (figure 4). Chez les deux races, la motilité massale n'a pas varié significativement avec l'âge ( $\mathrm{p}>0,05)$.

\section{Concentration en spermatozoüdes du sperme}

La saison a influencé de façon significative la concentration en spermatozoïdes du sperme des béliers Peuls $(p<0,05)$. La concentration la plus élevée a été enregistrée en avril et les plus faibles en août, octobre, novembre et décembre. Chez les béliers Touaregs, la saison n'a pas influencé significativement la concentration du sperme en spermatozoïdes ( $\mathrm{p}>0,05$ ) (figure 5).

Chez les béliers Peuls, la concentration en spermatozoïdes du sperme n'a pas varié significativement avec l'âge des animaux ( $p>0,05)$. En revanche, chez les béliers Touaregs la concentration a été significativement plus élevée $(\mathrm{p}<0,05)$ en 1996 par rapport aux années 1997 et 1998 (5 176 x 106 $\mathrm{spz} / \mathrm{ml}$ en 1996 contre $4828 \times 10^{6} \mathrm{spz} / \mathrm{ml}$ en 1997 et $4850 \times 10^{6} \mathrm{spz} / \mathrm{ml}$ en 1998).

\section{Nombre moyen de spermatozoïdes totaux}

La saison a influencé significativement la production spermatique chez les deux races $(\mathrm{p}<0,05)$. Le mois d'avril a été à la période de production maximale. La production a baissé significativement en juillet, août et décembre pour les béliers Peuls, et en octobre, décembre, janvier et juin pour les béliers Touaregs (figure 6). Comme pour le volume de sperme, en juillet-août les béliers Touaregs ont produit un nombre significativement plus élevé $(p<0,05)$ de spermatozoïdes que les béliers Peuls (respectivement 8556 $\times 10^{6} \pm 2328 \times 10^{6} \mathrm{spz}$ et $10849 \times 10^{6} \pm 2838 \times 10^{6} \mathrm{spz}$, contre $5883 \times 10^{6} \pm 1875 \times 10^{6} \mathrm{spz}$ et $6158 \times 10^{6} \pm 1963 \times 10^{6} \mathrm{spz}$ ).

Chez les deux races, le nombre moyen de spermatozoïdes totaux a été significativement plus élevé en 1997 ( $\mathrm{p}<0,05$ ), par rapport aux productions de 1996 et 1998. Chez les béliers Peuls, il a été obtenu $8158 \times 10^{6} \mathrm{spz}$ en 1997 contre $6941 \times 10^{6} \mathrm{spz}$ en 1996 et 7212

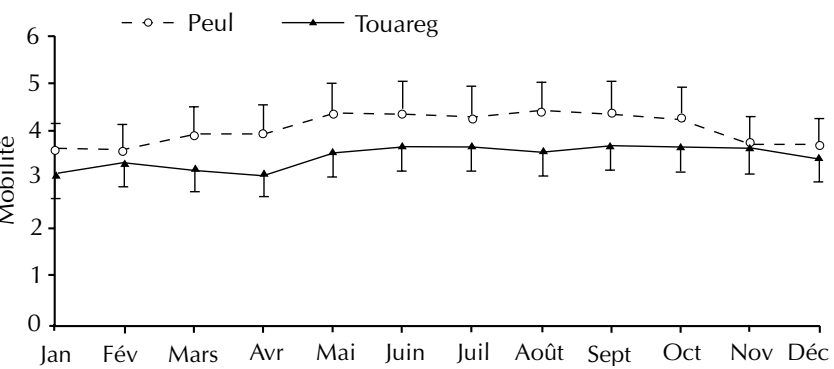

Figure 4 : variations mensuelles de la motilité massale moyenne chez les deux races de béliers (moyenne \pm écarttype).

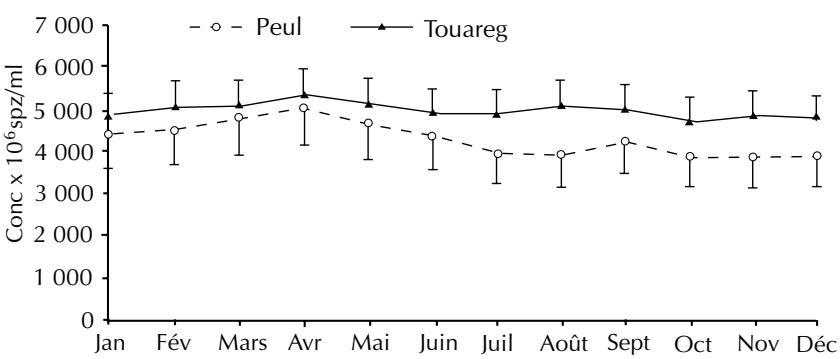

Figure 5 : variations mensuelles de la concentration moyenne du sperme chez les deux races de béliers (moyenne \pm écart-type). x $10^{6} \mathrm{spz}$ en 1998. Chez les béliers Touaregs, la production a été de $9809 \times 10^{6} \mathrm{spz}$ en 1997 , contre $7987 \times 10^{6}$ spz en 1996 et $8634 \times 10^{6}$ spz en 1998.

\section{Pourcentage de spermatozoïdes morts}

La saison a influencé significativement l'apparition de spermatozoïdes morts chez les béliers Peuls. La saison sèche fraîche (novembre à février) a coïncidé avec une augmentation du taux de spermatozoïdes morts. Chez les béliers Touaregs, la saison n'a pas influencé de façon significative ce pourcentage (figure 7). Le taux de spermatozoïdes morts n'a pas varié significativement en fonction de l'âge chez les deux races ( $\mathrm{p}>0,05)$.

\section{Pourcentage d'anomalies totales}

La saison sèche fraîche (novembre à février) a influencé significativement $(p<0,01)$ chez les béliers Peuls le pourcentage de spermatozoïdes anormaux qui a augmenté. Les taux les plus faibles ont été notés en mars, mai, août et septembre. Chez les béliers Touaregs, la saison n'a pas influencé ce paramètre (figure 8). Chez les deux races, le taux d'anomalies totales n'a pas varié significativement en fonction de l'âge $(\mathrm{p}>0,05)$.

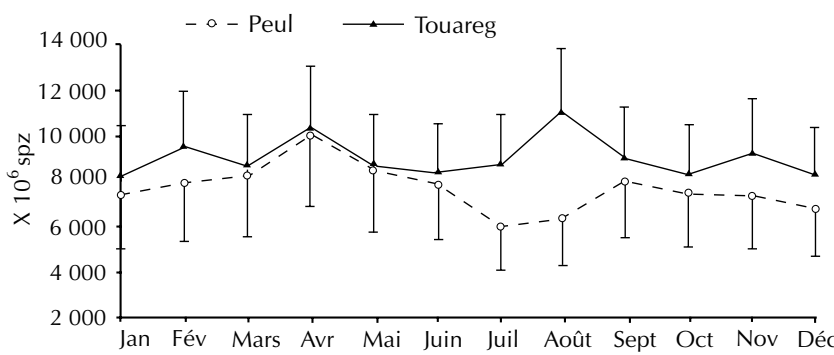

Figure 6 : variations mensuelles du nombre total moyen de spermatozoïdes chez les deux races de béliers (moyenne \pm écart-type).

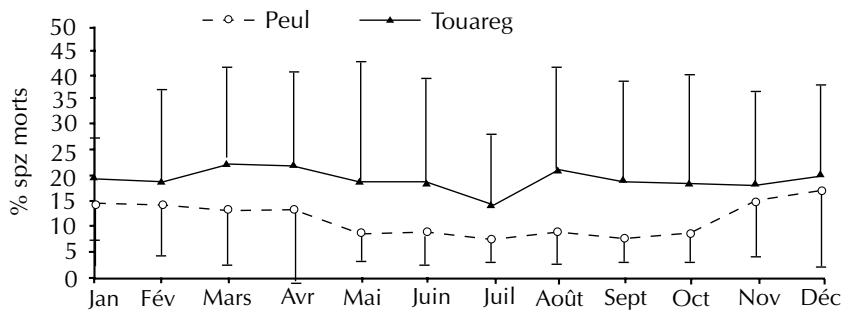

Figure 7 : variations mensuelles du taux moyen de spermatozoïdes morts chez les deux races de béliers (moyenne \pm écart-type).

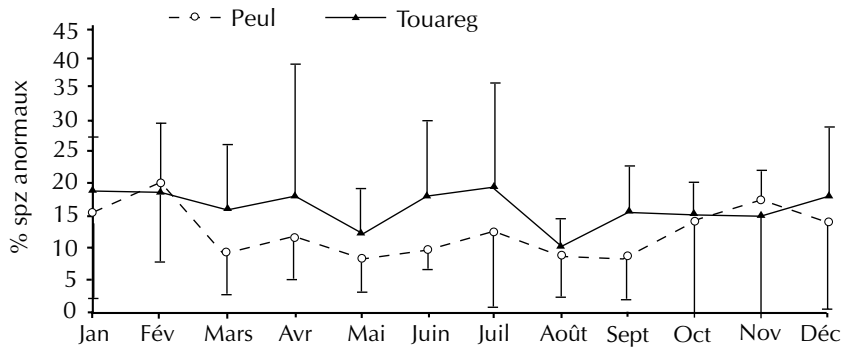

Figure 8 : variations mensuelles du taux moyen d'anomalies totales de spermatozoïdes chez les deux races de béliers (moyenne \pm écart-type). 


\section{DISCUSSION}

A l'issue des trois années d'expérimentation, le poids corporel moyen des béliers Touaregs a été plus élevé que celui des béliers Peuls. Cette différence de poids peut s'expliquer par la différence de poids moyen en début d'expérience. Elle est probablement d'origine génétique. Chez les deux races, il y a eu une variation saisonnière du poids corporel moyen. En effet, c'était pendant la saison sèche fraîche (novembre-décembre) que les animaux ont pris le plus de poids. Ces résultats confirment ceux obtenus chez des béliers de race Peule-peule du Sénégal (18). D'après ces études, la consommation de paille de riz, qui constituait la ration d'entretien des animaux, a été maximale en saison sèche fraîche (décembre à mars), mais l'élévation de la température à partir d'avril, conjuguée avec celle de l'hygrométrie, s'est accompagnée d'une baisse progressive de la consommation de paille de riz en saison sèche chaude, qui s'est poursuivie avec l'arrivée des pluies en juillet. Ce mécanisme peut donc expliquer la chute de poids observée dans la présente étude chez les deux races en période hivernale (juillet-août). Etant donné la relation très étroite qui existe entre le développement corporel et celui des testicules, cette chute de poids observée en juillet-août peut s'accompagner d'une diminution du poids et du diamètre testiculaire, comme cela a été observé chez certaines races européennes à certaines périodes de l'année (6)

Les volumes moyens de sperme après deux éjaculations (1,73 et $1,78 \mathrm{ml}$ respectivement chez les béliers Peuls bicolores et Touaregs) ont été comparables (1,78 ml) à ceux obtenus par d'autres auteurs (14) chez la race Touareg dans les mêmes conditions. Avec deux éjaculations, le volume de sperme collecté chez les béliers Peuls et Touaregs a été supérieur à celui collecté chez des jeunes béliers Romanov de 11 à 17 mois $(0,89 \mathrm{ml}$, deux séances par semaine avec deux éjaculats par séance de collecte) (7). Cette différence avec une race européenne peut être liée au rythme de collecte, à l'âge, au poids et à l'alimentation des animaux. Le volume de sperme étant corrélé au poids corporel $(7,8,17)$, la baisse du volume de sperme qui a été observée chez la race Peule en juillet et août pourrait s'expliquer par la chute du poids corporel et des testicules consécutive aux taux élevés d'humidité relative et à une réduction de la prise alimentaire $(6,18)$. Chez la race Touareg, les variations saisonnières n'ont pas influencé significativement le volume de sperme éjaculé, probablement en raison d'une perte de poids par unité de temps moins importante que pour la race Peule (respectivement $-1,3$ p. 100 contre $-1,6$ p. 100 de poids vif par mois).

La motilité du sperme des béliers Peuls bicolores a été supérieure à celle des béliers Touaregs (4 contre 3 ). Cette observation corrobore les résultats relevés chez les béliers Peuls blancs $(3,2)$ et Touaregs $(3,1)(14)$. En milieu tropical nigérian, on n'observe pas de différence de motilité du sperme entre les races Udda, Balami et Yankassa (collecté une fois par semaine avec deux éjaculats par séance) (12). En milieu européen, on observe que les béliers de race Dorset Horn ont une meilleure motilité massale que les béliers Mérinos, Texel et Suffolk $(2,13)$. La baisse de la motilité massale de décembre à février chez les deux races de béliers étudiées ici peut s'expliquer par une faible maturation épididymaire (10) ou par les effets négatifs des amplitudes thermiques journalières élevées et les taux d'humidité faibles $(13,16)$ qui caractérisent les mois de décembre à avril au Sahel. Etant donné la relation qui existe entre la motilité massale et la fertilité du troupeau $(3,8)$ et les résultats obtenus dans la présente étude, il semble que les béliers Peuls et Touaregs sont potentiellement fertiles toute l'année dans les conditions expérimentales (bon suivi en station).
Les concentrations moyennes de spermatozoïdes obtenues dans la présente étude par race durant les trois années de l'expérimentation (4 $265 \times 10^{6} \mathrm{spz} / \mathrm{ml}$ pour les béliers Peuls et $4953 \times 10^{6} \mathrm{spz} / \mathrm{ml}$ pour les béliers Touaregs) ont été supérieures à celles rapportées chez les béliers Peuls blancs et Touaregs (respectivement $3694 \times 10^{6} \mathrm{spz} / \mathrm{ml}$ et $3581 \times 10^{6} \mathrm{spz} / \mathrm{ml}$ ) (14). Ceci peut s'expliquer par une différence de période de collecte du sperme entre les deux expériences. Les résultats de la présente étude pour les béliers Peuls bicolores ont été comparables à ceux de Harouna pour la même race $\left(3990 \times 10^{6} \mathrm{spz} / \mathrm{ml}\right.$ à $5020 \times 10^{6} \mathrm{spz} / \mathrm{ml}$; deux éjaculats par séance de collecte) (11) et à ceux d'Osinowo et coll. pour les béliers Yankassa du Nigeria, avec une collecte une fois par jour du 1 er au 14 juillet (3 $421 \times 10^{6}$ à $4673 \times 10^{6} \mathrm{spz} / \mathrm{ml}$ ) (17). Les béliers Touaregs ont eu une concentration de spermatozoïdes comparable à celle de jeunes béliers Romanov, race européenne très prolifique $\left(5020 \times 10^{6} \mathrm{spz} / \mathrm{ml}\right.$; deux séances de collecte par semaine avec deux éjaculats par séance) (7). Les variations raciales enregistrées peuvent être d'origine génétique ou liées à des facteurs environnementaux et des conditions d'élevage très différentes qui caractérisent les deux systèmes écologiques où vivent ces deux races de moutons.

La chute de poids des animaux durant la saison hivernale a été probablement responsable chez la race Peule bicolore de la baisse de concentration du sperme observée en août. La baisse de la concentration en spermatozoïdes du sperme qui a été observée d'octobre à décembre pouvait être liée, quant à elle, à l'accroissement de l'amplitude thermique journalière constaté au cours de l'année dans cette région. Cette action de la température se ferait par l'intermédiaire de l'hormone LH dont le rythme est modifié (22). Pour les béliers Touaregs, les variations des facteurs climatiques n'ont pas influencé de manière significative la concentration spermatogénétique ; ceci pouvait être le résultat du transfert de ces animaux du nord au sud où les variations des facteurs climatiques sont beaucoup moins marquées qu'au nord.

La production totale de spermatozoïdes par récolte a été variable selon la race, le bélier Touareg ayant eu une production significativement plus importante que le bélier Peul bicolore (respectivement $8877 \times 10^{6} \mathrm{spz}$ contre $7469 \times 10^{6} \mathrm{spz}$ ). Il est probable que la multiplication des cellules spermatiques soit plus importante chez le bélier Touareg que chez le bélier Peul bicolore. Ceci peut être rapproché des travaux chez la brebis Touareg (1) et la brebis Peule bicolore (23) où il a été montré que l'index mitotique des follicules ovariens est plus grand chez la race Touareg que chez la race Peule bicolore. Compte tenu du fait que la multiplication des cellules germinales et leur développement sont contrôlés par les mêmes types d'hormones chez le mâle et la femelle, on peut émettre l'hypothèse de l'existence d'un index mitotique plus grand des spermatogonies et des spermatocytes chez la race Touareg que chez la race Peule. Toutefois, cette hypothèse devrait être confirmée par une étude du cycle spermatogénétique des deux races.

Les différents pourcentages moyens de spermatozoïdes morts relevés respectivement chez les béliers Peuls bicolores et Touaregs (12 et 19 p. 100) n'ont pas été élevés par rapport aux limites acceptables pour un bon géniteur ovin (20 à 30 p. 100) (5). Cependant, il y a eu de grandes variations individuelles, surtout chez la race Touareg où 21,7 p. 100 des éjaculats ont eu des taux d'anomalies supérieurs à 30 p. 100. Chez cette dernière, le taux de spermatozoïdes morts n'a pas subi de variation saisonnière. En revanche, chez les béliers Peuls, la période allant de novembre à février a coïncidé avec des taux plus élevés de spermatozoïdes morts $(6,5$ p. 100 des éjaculats ont eu des taux supérieurs à 30 p. 100). C'est au cours de cette période que les écarts thermiques ont été élevés. 
Les taux de spermatozoïdes anormaux (13 et 17 p. 100), relevés respectivement chez les béliers Peuls bicolores et Touaregs, se sont situés dans les limites acceptables pour obtenir une bonne fertilité. En effet, une étude réalisée sur des ovins de race Ile-de-France a montré des taux de fertilité du troupeau de 50 à 60 p. 100 quand le pourcentage de spermatozoïdes anormaux est de 10 à 20 p. 100 et de moins de 50 p. 100 quand le pourcentage de spermatozoïdes anormaux est de 40 à 60 p. 100 (5). Chez la race Touareg, 27 et 4,6 p. 100 des éjaculats ont eu respectivement des taux d'anomalies supérieurs à 20 et 40 p. 100 ; ce paramètre n'a pas subi de variations saisonnières. Chez la race Peule bicolore, la période allant de novembre à février a coïncidé avec des taux élevés de spermatozoïdes anormaux $(15,1$ et 2,5 p. 100 des éjaculats ont eu respectivement des taux d'anomalies supérieurs à 20 et 40 p. 100), résultant de défauts spermatogénétiques ou de maturation épididymaire. C'est au cours de cette même période qu'ont été enregistrés pour la race Peule des pourcentages élevés de spermatozoïdes morts, impliquant probablement le même mécanisme pour les deux types de spermatozoïdes $(4,6)$.

\section{BIBLIOGRAPHIE}

1. BANOIN M., MARIANA J.C., YENIKOYE A., 1991. Folliculogenèse autour de l'œstrus chez les brebis Touaregs nigériennes. In : Journées scientifiques de l'aupelf/Uref, Dakar, Sénégal, 9-11 juin 1991.

2. BOLAND M.P. AL-KAMALI A.A., CROSBY T.F., HAYNES N.B. HOWLES C.M., KELLEHER D.L., GORDON I., 1985. The influence of breed season and photoperiod on semen characteristics, testicular size, libido and plasma hormone concentrations in rams. Anim. Reprod. Sci., 9: $241-252$

3. COLAS G., 1983. Factors affecting the quality of ram semen. In: Sheep production, 1st Edn. Nottingham, England, Butterworths, p. 453-465.

4. COLAS G., GUERIN Y., 1980. Variations saisonnières de la qualité du sperme chez le bélier Ile-de-France. I. Etude de la morphologie cellulaire et de la motilité massale. Reprod. Nutr. Dév., 20 : 1789-1799.

5. COLAS G., GUERIN Y., 1981. Variation saisonnière de la qualité du sperme chez le bélier lle-de-France. II. Fécondance : relation avec les critères qualitatifs observés in vitro. Reprod. Nutr. Dév., 21 : 399-407.

6. COLAS G., GUERIN Y., LEMAIRE Y., MONTASSIER Y., DESPIERRES J., 1986. Variations saisonnières du diamètre testiculaire et de la morphologie des spermatozoïdes chez le bélier Vendéen et chez le bélier Texel. Reprod. Nutr. Dév., 26 : 863-875.

7. COLAS G., PERSONNIC D., COUROT M., ORTAVANT R., 1975. Influence du rythme de récolte sur la production de spermatozoïdes chez le jeune bélier Romanov. Ann. Zootech., 2 : 189-198.

8. CORTEEL J.M., 1994. La reproduction du mâle de I'espèce caprine. In : $9^{e}$ réunion nationale sur l'élevage caprin, La Paz, Basse Californie, Bcs, Mexique, 27-30 septembre 1994, 24 p.

9. DICKO M.S., SAYERS R., 1988. Recherches sur le système agropastoral de production de la zone semi-aride du Niger : Etude de la composante animale. Niamey, Niger, Cipea, 148 p.

10. FOURNIER DELPECH S., COLAS G., COUROT M., ORTAVANT R., 1979. Epididymal sperm maturation in the ram: motility, fertilizing ability and embryonic survival after uterine artificial insemination in the ewe. Ann. Biol. Anim. Biochem. Biophys., 19: 597-605.

11. HAROUNA A., 1987. Etude de quelques caractéristiques morphologiques du sperme de bélier Peul bicolore du Niger. Rapport de stage. Niamey, Niger, faculté d'agronomie, $15 \mathrm{p}$.

12. KUMI-DIAKA J., ADESIYUN A.A., SEKONI V., EZEOKOLI C.D., 1985. Scrotal dimensions and ejaculate characteristics of three breeds of sheep in tropical Nigeria. Theriogenology, 23: 671-677.

13. LINDSAY D.R., 1969. Sexual activity and semen production of rams at high temperatures. J. Reprod. Fert., 18: 1-8.

\section{CONCLUSION}

Au regard de tous ces paramètres morphologiques (motilité massale, concentration, mortalité et anomalie), dans les conditions expérimentales de cette étude, la qualité du sperme des béliers Peuls et Touaregs est restée bonne toute l'année. Ceci est probablement l'expression du caractère d'adaptation des deux races au climat sahélien difficile, mais aussi des bonnes conditions d'entretien des animaux dans ce travail réalisé en station. Les résultats ont aussi montré que le climat sahélien influence peu la spermatogenèse chez le bélier. Ceci n'a pas été le cas chez des brebis des deux races élevées en station où au moins 52 p. 100 des individus ont eu une baisse de leur activité sexuelle au cours de la période décembre-avril marquée par des amplitudes thermiques circadiennes importantes $(20,21)$. S'agissaitil alors d'individus non sensibles aux variations thermiques saisonnières au niveau de la spermatogenèse ? Il serait utile de connaître ultérieurement, dans une perspective de recherche-développement, l'impact des conditions d'élevage traditionnel, de la saison et de l'individu sur les paramètres de fertilité des mâles et des femelles, et donc sur la productivité numérique du troupeau.

14. MARICHATOU $\mathrm{H}$, YENIKOYE A, BANOIN M, 1993. Etude de quelques caractéristiques morphologiques du sperme de béliers Peuls blancs et Touaregs. In : Bourzat D. Ed. sci., Actes du Comité scientifique de Garoua, Cameroun, 15-20 février 1993. Montpellier, France, Cirademvt, p. 33-41.

15. MARICHATOU H., YENIKOYE A., BANOIN M., 1994. Quelques données sur le sperme de béliers Peuls blancs et Touaregs du Niger. In: Proc. 3rd Biennal Conference of the African Small Ruminant Research Network UICC, Kampala, Uganda, 5-9 December 1994.

16. NIELSEN M., GALET M., SYMOENS C., HARDOUIN J., 1985. Note sur la stérilisation provoquée du bélier en milieu tropical. Revue. Elev. Méd. vét. Pays trop., 38 : 195-199.

17. OSINOWO O.A., AHMED M.S., EKPE G.A., 1988. Semen quality and sperm output of Yankasa rams at different ages. Theriogenology, 29: $381-386$

18. SALL C., GUERIN N., AHOKPE B., FRET D., 1987. Les variations saisonnières de la capacité d'ingestion des moutons en zone tropicale sèche. Reprod. Nutr. Dév., 27 : 203-204.

19. SNEDECOR G.W., COCHRAN W.G., 1957. Statistical methods. Ames, IA, USA, University Press, $649 \mathrm{p}$.

20. TOUKOU Y., BANOIN M., YENIKOYE A., MARICHATOU H., HASSANE M., 1994. Variations saisonnières du comportement d'œstrus et détermination du moment de l'ovulation sur œestrus induit et œestrus naturel chez deux races de brebis nigériennes : la race Touareg et la race Peule blanche. In: Proc. Regional Seminar of the International Foundation for Science (IFS), Niamey, Niger, 17-21 January, p. 141-158.

21. YENIKOYE A., 1984. Variations annuelles du comportement d'œstrus, du taux et des possibilités d'ovulation chez la brebis Peule du Niger. Reprod. Nutr. Dév., 24 : 11-19.

22. YENIKOYE A., 1986. Etudes de l'endocrinologie sexuelle et de la croissance folliculaire chez la brebis nigérienne de race Peulh : influence de la saison de reproduction. Thèse Doct. Etat Sciences naturelles, université de Tours, France, 91 p.

23. YENIKOYE A., MARIANA J.C., 1990. Population de follicules ovariens chez la brebis Peule. Revue Elev. Méd. vét. Pays trop., $43: 243-248$.

24. YENIKOYE A., MARICHATOU H., 1993. Productivité et activité ovarienne de la brebis Peule dans le système d'élevage traditionnel au Niger. Rome, Italie, Fao / Vienne, Autriche, laea / Cachan, France, Tec\&Doc, 708, p. 133-142.

Reçu le 03.09.2001, accepté le 07.06.2002 


\section{Summary}

Issa M., Yenikoye A., Marichatou H., Banoin M. Spermogram of Bicolor Peul and Touareg Rams: Genetic Type and Seasonal Influence

A study on the morphological parameters of ram sperm was carried out in an experimental station in Niger during three years. Six bicolor Peul rams and five Touareg rams were used. Sperm from each ram was collected after two consecutive ejaculations once a month with an artificial vagina. Means $( \pm$ SE) of the various parameters studied in Peul and Touareg rams were, respectively: live weight $47.75 \pm 3.75 \mathrm{~kg}$ and $49.98 \pm 2.99 \mathrm{~kg}$; sperm volume $1.73 \pm 0.36 \mathrm{ml}$ and $1.78 \pm 0.33 \mathrm{ml}$; sperm gross motility $4 \pm 0.61$ and $3 \pm 0.49$; spermatozoon concentration $4265 \times 10^{6} \pm 762 \times 10^{6} \mathrm{spz} / \mathrm{ml}$ and $4953 \times 10^{6} \pm 583 \times 10^{6} \mathrm{spz} / \mathrm{ml}$; total number of spermatozoa $7469 \times 10^{6} \pm 2382 \times 10^{6} \mathrm{spz}$ and $8877 \times 10^{6}$ $\pm 2323 \times 10^{6} \mathrm{spz}$; percentage of dead spermatozoa $12 \pm 10 \%$ and $19 \pm 19 \%$; percentage of abnormal spermatozoa 13 $\pm 12 \%$ and $17 \pm 11 \%$. The mean weight of Touareg rams was significantly higher $(p<0.05)$ than that of Peuls. The dry cool season significantly $(p<0.05)$ and negatively affected the live weights of both breeds. Mean volumes of ejaculated sperm were not significantly $(p<0.05)$ different between the two breeds, except during the months of July and August. Significant $(p<0.05)$ differences were found between the two breeds with regard to the other parameters studied. Peul rams appeared more sensitive to Sahelian climate variations than Touaregs. Under the experimental conditions of the study (i.e. in station and under good management), ram fertility of both breeds was maintained in line with the established norms throughout the year, thus suggesting that rams might be mated with ewes year-round. Further studies would help determine the impact of traditional breeding conditions, season, individual characteristics on male and female fertility parameters, and consequently on the flock size.

Key words: Peul sheep - Touareg sheep - Semen - Fertility Niger.

\section{Resumen}

Issa M., Yenikoye A., Marichatou H., Banoin M. Espermiograma en carneros Peul bicolores y Tuareg: influencia del tipo genético y de la estación

Se estudiaron los parámetros morfológicos del esperma en la estación experimental en seis carneros de raza Peul bicolor y cinco carneros de raza Targui de Níger, durante tres años consecutivos. El esperma fue recolectado con vagina artificial una vez por mes y por carnero, después de dos eyaculaciones por episodio. Los promedios (mas o menos la desviación estándar) obtenidos para los diferentes parámetros estudiados fueron respectivamente en los carneros Peul y Tuareg: peso vivo $47,75 \pm 3,75 \mathrm{~kg}$ y $49,98 \pm 2,99 \mathrm{~kg}$, volumen de esperma $1,73 \pm 0,36 \mathrm{ml}$ y $1,78 \pm 0,33 \mathrm{ml}$, motilidad en masa $4 \pm 0,61$ y $3 \pm 0,49$, concentración de espermatozoides $4265 \times 10^{6}$ $\pm 762 \times 10^{6} \mathrm{spz} / \mathrm{ml}$ y $4953 \times 10^{6} \pm 583 \times 10^{6} \mathrm{spz} / \mathrm{ml}$, número total de espermatozoides $7469 \times 10^{6} \pm 2382 \times 10^{6} \mathrm{spz}$ y 8877 $\times 10^{6} \pm 2323 \times 10^{6} \mathrm{spz}$, porcentaje de espermatozoides muertos $12 \pm 10 \%$ y $19 \pm 19 \%$, porcentaje de espermatozoides anormales $13 \pm 12 \%$ y $17 \pm 11 \%$. El peso de los carneros Tuareg fue mayor que el de los carneros Peul $(p<0,05)$. En las dos razas la estación seca y fresca afectó de manera significativa y negativa el peso vivo $(p<0,05)$. El volumen medio de esperma eyaculado no fue diferente entre las dos razas, excepto durante los meses de julio y agosto $(p<0,05)$. Para los otros parámetros estudiados, se encontraron diferencias significativas entre las razas $(p<0,05)$. Los carneros Peul parecen más sensibles a las variaciones del clima sahariano que los carneros Tuareg. Bajo las condiciones experimentales del presente trabajo (buen seguimiento en la estación), la fertilidad de los carneros de las dos razas se mantuvo bien todo el año, con relación a las normas requeridas. Esto indica una posibilidad de salto a las hembras todo el año. Posteriormente, sería de utilidad conocer el impacto de las condiciones de la cría tradicional, de la estación y del individuo sobre los parámetros de fertilidad de los machos y de las hembras y por ende sobre la productividad numérica del hato.

Palabras clave: Ovino Peul - Ovino Tuareg - Semen - Fertilidad - Niger. 